\title{
LOCAL TENSOR RADIATION CONDITIONS FOR ELASTIC WAVES
}

\author{
S. KRENK \\ Department of Civil Engineering, Technical University of Denmark, DK-2800 Lyngby, \\ Denmark.E-mail:sk@bkm.dtu.dk \\ AND \\ P. H. KiRKEGAARD \\ Department of Civil Engineering, Aalborg University, Sohngaardsholmsvej 57, \\ DK-9000 Aalborg, Denmark
}

(Received 22 May 2000, and in final form 9 May 2001)

\begin{abstract}
A local boundary condition is formulated, representing radiation of elastic waves from an arbitrary point source. The boundary condition takes the form of a tensor relation between the stress at a point on an arbitrarily oriented section and the velocity and displacement vectors at the point. The tensor relation generalizes the traditional normal incidence impedance condition by accounting for the angle between wave propagation and the surface normal and by including a generalized stiffness term due to spreading of the waves. The effectiveness of the local tensor radiation condition is demonstrated by detailed finite element time and frequency analysis of a concentrated force in infinite three-dimensional space, and by a time analysis of a pulse load in a two-dimensional underground gallery.
\end{abstract}

(C) 2001 Academic Press

\section{INTRODUCTION}

Many problems of elastic wave propagation involve infinite domains, and the solution of such problems by finite element or finite difference methods must therefore account for the existence of an infinite medium beyond the finite part included in the mesh and bounded by a radiation boundary, introduced in the model; see Figure 1. Different methods exist for representing the influence of the infinite medium beyond the radiation boundary such as infinite elements, coupling to a boundary element representation of the infinite domain, and the use of radiation boundary conditions.

The elastic wave propagation problem is governed by the equations of motion

$$
\boldsymbol{\nabla} \cdot \boldsymbol{\sigma}-\rho \ddot{\mathbf{u}}+\mathbf{f}=\mathbf{0},
$$

where $\boldsymbol{\sigma}$ is the stress tensor, $\mathbf{u}$ the displacement vector, and $\rho$ the mass density. $\boldsymbol{\nabla}$ is the gradient or divergence operator and an overdot indicates time differentiation. In a variational setting, the form of the boundary conditions follows from multiplication of the field equations by a virtual displacement vector $\tilde{\mathbf{u}}$, integration over the material volume, and use of the divergence theorem. The result is

$$
\int_{S} \tilde{\mathbf{u}} \cdot(\hat{\mathbf{n}} \cdot \boldsymbol{\sigma}) \mathrm{d} S-\int_{V}(\tilde{\boldsymbol{\varepsilon}}: \boldsymbol{\sigma}+\rho \tilde{\mathbf{u}} \cdot \ddot{\mathbf{u}}-\tilde{\mathbf{u}} \cdot \mathbf{f}) \mathrm{d} V=0 .
$$




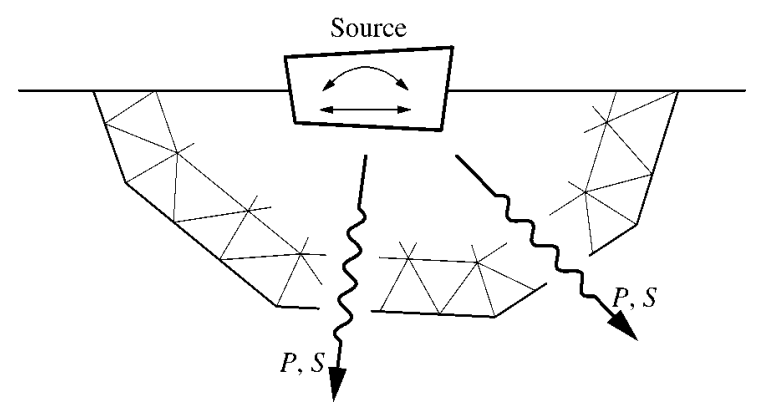

Figure 1. Radiation or scattering of elastic waves.

The boundary conditions are identified from the surface integral in the first term. The traditional boundary conditions are the prescribed displacement vector, whereby $\tilde{\mathbf{u}}=\mathbf{0}$, or a prescribed boundary stress vector $\tau=\hat{\mathbf{n}} \cdot \boldsymbol{\sigma}$. In both these cases, the variational surface integral can be given a value. Within a variational formulation, a radiation boundary condition must similarly enable the evaluation of the contribution to the boundary integral from the radiation boundary. This amounts to the establishment of an expression for the boundary stress vector $\tau$ in terms of the displacement and velocity vector fields $\mathbf{u}$ and $\dot{\mathbf{u}}$, representing radiation from the boundary.

Strictly speaking, the radiation condition involves the full time history of the displacement field up to the current time, and while this can in principle be included via a boundary element representation of the exterior problem, that would severely complicate the solution. Therefore, several alternatives have been used. The simplest of these is the use of isolated dampers, simulating an impedance boundary condition $[1,2]$. A recent survey of this type of boundary condition and some generalizations has been given by Kellezi [3], who also suggested the use of a spring term to represent spreading of the waves. Improved solutions can be obtained by introducing non-local boundary conditions, that account for the variation of the wave field. A procedure of this type in which the exterior problem is represented by a self-similar sequence of finite elements has been devised by Wolf and Song [4], and further developed by Paronesso and Wolf [5], who obtained a rational function representation for the delayed effects and recast the problem into an extended system of equations with the memory represented indirectly via internal variables associated with the exterior problem.

It is a characteristic of these references, that the radiation boundary conditions are constructed after the discretization of the field equations. A survey of local boundary conditions for various wave propagation problems including acoustic and elastic waves has been given by Givoli [6]. In the present paper, the basis of local radiation boundary conditions is reconsidered, and a set of tensor relations are derived that account consistently for inclined incidence and finite distance of the radiating source from the boundary. In cases where this information is available, the local tensor boundary conditions constitute a simple means of representing the infinite medium beyond the radiation boundary, generalizing the scalar impedance boundary conditions of Lysmer and Kuhlemeyer [1] to non-normal incidence and diverging waves.

Improved boundary conditions can be developed by elimination of the explicit dependence on the angle of incidence [6] and recent work has led to a formulation of higher order radiation boundary conditions for the acoustic problem in accordance with variational principles, and therefore compatible with conventional finite element formulation [7]. An extension of this formulation to elastic waves is in progress, but the 

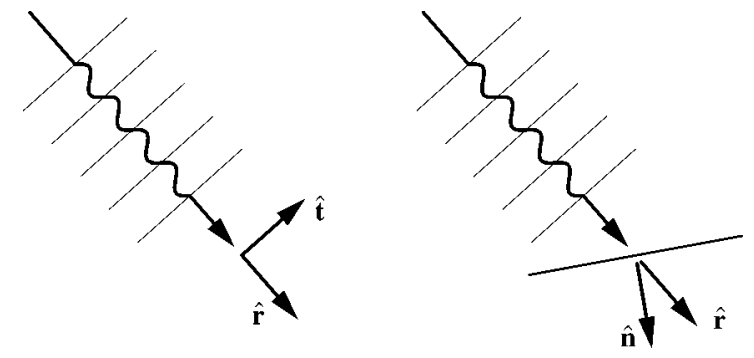

Figure 2. Plane elastic wave field propagating in direction $\hat{\mathbf{r}}$.

elastic wave problem is complicated by the tensor character of the equations and the existence of surface waves. In principle, ideal radiating boundary conditions can be formulated if an explicit representation of the exterior problem is available, see e.g., reference [8], but in elastodynamics surface and interface waves prevent simple representations except for the ideal scattering problem in an infinite homogeneous medium.

\section{IMPEDANCE TENSOR FOR PLANE WAVES}

Figure 2(a) illustrates a plane elastic wave field propagating in the direction of the unit vector $\hat{\mathbf{r}}$. The corresponding displacement field can be written as the sum of a primary compression wave field $\mathbf{u}_{P}$ and a secondary shear wave field $\mathbf{u}_{S}$,

$$
\mathbf{u}=\mathbf{u}_{P}+\mathbf{u}_{S} .
$$

The primary wave field consists of displacements in the direction $\hat{\mathbf{r}}$, propagating with wave speed $c_{P}$

$$
\mathbf{u}_{P}=\hat{\mathbf{r}} u_{P}\left(t-r / c_{P}\right)
$$

The secondary wave consists of transverse motion, here assumed to be in the transverse direction defined by the unit vector $\hat{\mathbf{t}}$, propagating with wave speed $c_{S}$,

$$
\mathbf{u}_{S}=\hat{\mathbf{t}} u_{S}\left(t-r / c_{S}\right)
$$

The particular form (4) and (5) of the primary and secondary wave fields leads to the plane wave identities,

and

$$
\left(\frac{\partial}{\partial t}+c_{P} \frac{\partial}{\partial r}\right) \mathbf{u}_{P}=\mathbf{0}
$$

$$
\left(\frac{\partial}{\partial t}+c_{S} \frac{\partial}{\partial r}\right) \mathbf{u}_{S}=\mathbf{0} .
$$

These identities contain the information to be expressed in the so-called radiation condition.

The radiation condition for plane elastic waves is now expressed as a tensor relation between the stress vector on an arbitrarily oriented section with outward normal vector $\hat{\mathbf{n}}$, Figure 2(b), and the velocity vector $\dot{\mathbf{u}}$. The stress contributions from the compression and shear waves are derived separately and then combined to yield the radiation condition as a tensor relation between the total stress vector and the total velocity. 


\subsection{PLANE P-WAVES}

The strain field corresponding to the plane P-wave (4) with axial displacement $u_{P}=\hat{\mathbf{r}} \cdot \mathbf{u}_{P}$ consists of the uniaxial strain $\varepsilon_{r}=\partial u_{P} / \partial r$. The strain tensor of the P-wave field then is

$$
\varepsilon_{P}=\hat{\mathbf{r}} \hat{\mathbf{r}} \frac{\partial u_{P}}{\partial r}=-\hat{\mathbf{r}} \hat{\mathbf{r}} \frac{1}{c_{P}} \dot{u}_{P}
$$

where the last result follows from P-wave identity (6). Here and in the following, a group of two vectors like $\hat{\mathbf{r}} \hat{\mathbf{r}}$, without an operator like the dot denoting scalar product, designates the vector dyad; see, e.g., Appendix A.

The uniaxial strain field can be decomposed into two parts, representing isotropic compression and deviatoric strain, respectively,

$$
\varepsilon_{P}=-\left[\frac{1}{3} \mathbf{1}+\left(\hat{\mathbf{r}} \hat{\mathbf{r}}-\frac{1}{3} \mathbf{1}\right)\right] \frac{1}{c_{P}} \dot{u}_{P},
$$

where 1 denotes the second order unit tensor.

For an isotropic elastic material, the stresses follow from the strains by multiplying the isotropic strain components by $3 k$, and the deviatoric strains by $2 \mu$, where $k$ is the bulk modulus and $\mu$ is the shear modulus. The bulk modulus is given in terms of the Lamé parameters $\lambda$ and $\mu$ as

$$
k=\lambda+\frac{2}{3} \mu .
$$

The stress field then follows directly from equations (9) and (10) as

$$
\boldsymbol{\sigma}_{P}=-\left[k \mathbf{1}+2 \mu\left(\hat{\mathbf{r}} \hat{\mathbf{r}}-\frac{1}{3} \mathbf{1}\right)\right] \frac{1}{c_{P}} \dot{u}_{P}=-[\lambda \mathbf{1}+2 \mu \hat{\mathbf{r}} \hat{\mathbf{r}}] \frac{1}{c_{P}} \dot{u}_{P} .
$$

It is seen that the isotropic part of the stress field contains transverse components, proportional to $\lambda$.

The stress vector on a section with unit normal vector $\hat{\mathbf{n}}$ follows from stress tensor (11) as

$$
\tau_{P}=\hat{\mathbf{n}} \cdot \boldsymbol{\sigma}=-[\lambda \hat{\mathbf{n}}+2 \mu(\hat{\mathbf{n}} \cdot \hat{\mathbf{r}}) \hat{\mathbf{r}}] \frac{1}{c_{P}} \dot{u}_{P} .
$$

When the magnitude of the P-wave velocity is introduced via $\dot{u}_{P}=\hat{\mathbf{r}} \cdot \dot{\mathbf{u}}$, stress vector relation (12) takes the form

$$
\tau_{P}=-\mathbf{Z}_{P} \cdot \dot{\mathbf{u}}
$$

where the impedance tensor $\mathbf{Z}_{P}$ for the $\mathrm{P}$-wave is

$$
\mathbf{Z}_{P}=\frac{1}{c_{P}}[\lambda \hat{\mathbf{n}} \hat{\mathbf{r}}+2 \mu(\hat{\mathbf{n}} \cdot \hat{\mathbf{r}}) \hat{\mathbf{r}} \hat{\mathbf{r}}]
$$

The impedance tensor $\mathbf{Z}_{P}$ gives the stress vector $\tau_{P}$ associated with a plane compression wave propagating in the direction $\hat{\mathbf{r}}$ in terms of the total velocity vector $\dot{\mathbf{u}}$. Its role is similar to the scalar impedance of acoustic waves in a fluid; see, e.g., Pierce [11]. However, the vectorial nature of the elastic wave propagation problem leads to tensor character of the impedance, and to explicit dependence on the normal $\hat{\mathbf{n}}$ of the section on which the stress vector acts. 


\subsection{PLANE S-WAVES}

The plane shear wave field (5) is associated with transverse displacements of magnitude $u_{S}$. The corresponding strain tensor is

$$
\varepsilon_{S}=\frac{1}{2}(\hat{\mathbf{t}} \hat{\mathbf{r}}+\hat{\mathbf{r}} \hat{\mathbf{t}}) \frac{\partial u_{S}}{\partial r}=-\frac{1}{2}(\hat{\mathbf{t}} \hat{\mathbf{r}}+\hat{\mathbf{r}} \hat{\mathbf{t}}) \frac{1}{c_{S}} \dot{u}_{S},
$$

where the last result follows from the S-wave identity (7).

The strain field is completely deviatoric, and thus the corresponding stress tensor follows from multiplication by $2 \mu$ :

$$
\boldsymbol{\sigma}_{S}=-\mu(\hat{\mathbf{t}} \hat{\mathbf{r}}+\hat{\mathbf{r}} \hat{\mathbf{t}}) \frac{1}{c_{S}} \dot{u}_{S}
$$

The stress vector on a section with unit normal $\hat{\mathbf{n}}$ is

$$
\tau_{S}=\hat{\mathbf{n}} \cdot \boldsymbol{\sigma}_{S}=-\mu[(\hat{\mathbf{n}} \cdot \hat{\mathbf{t}}) \hat{\mathbf{r}}+(\hat{\mathbf{n}} \cdot \hat{\mathbf{r}}) \hat{\mathbf{t}}] \frac{1}{c_{S}} \dot{u}_{S}
$$

When using the velocity vector of the shear wave field given as $\dot{\mathbf{u}}_{S}=\hat{\mathbf{t}} \dot{u}_{S}$, the stress vector (17) can be expressed in terms of the velocity vector $\dot{\mathbf{u}}_{S}$ in the form

$$
\tau_{S}=-\frac{\mu}{c_{S}}[\hat{\mathbf{r}} \hat{\mathbf{n}}+(\hat{\mathbf{n}} \cdot \hat{\mathbf{r}}) \mathbf{1}] \cdot \dot{\mathbf{u}}_{S}
$$

Note, that in this expression, the specific transverse direction defined by the unit vector $\hat{\mathbf{t}}$ does not appear explicitly, and thus the same relation applies to shear waves of any polarization relative to the normal vector $\hat{\mathbf{n}}$.

The final step in the derivation of the shear wave impedance formula is to extract the shear wave velocity vector $\dot{\mathbf{u}}_{S}$ from a general plane wave field containing both P- and $\mathrm{S}$-waves via the transverse projection $\dot{\mathbf{u}}_{S}=(\mathbf{1}-\hat{\mathbf{r}} \hat{\mathbf{r}}) \cdot \dot{\mathbf{u}}$. When this transverse projection is introduced into equation (18), the final relation is

$$
\tau_{S}=-\mathbf{Z}_{S} \cdot \dot{\mathbf{u}}
$$

where the impedance tensor $\mathbf{Z}_{S}$ for the $\mathrm{S}$-wave is

$$
\mathbf{Z}_{S}=\frac{\mu}{c_{S}}[\hat{\mathbf{r}} \hat{\mathbf{n}}+(\hat{\mathbf{n}} \cdot \hat{\mathbf{r}})(\mathbf{1}-2 \hat{\mathbf{r}} \hat{\mathbf{r}})]
$$

The impedance tensor $\mathbf{Z}_{S}$ extracts the stress vector associated with any plane shear wave field propagating in the direction $\hat{\mathbf{r}}$ and is not limited to plane polarization.

\subsection{GENERAL PLANE WAVE FIELD}

The total stress vector in a plane wave field containing both P- and S-waves is found by the addition of contributions (13) and (19):

$$
\tau=-\mathbf{Z} \cdot \dot{\mathbf{u}}
$$


The impedance tensor of a plane wave field propagating in the direction $\hat{\mathbf{r}}$ is given by the sum of equations (14) and (20),

$$
\mathbf{Z}=\mathbf{Z}_{P}+\mathbf{Z}_{S}=\frac{2 \mu}{c_{P}}(\hat{\mathbf{n}} \cdot \hat{\mathbf{r}}) \hat{\mathbf{r}} \hat{\mathbf{r}}+\frac{\mu}{c_{S}}(\hat{\mathbf{n}} \cdot \hat{\mathbf{r}})(\mathbf{1}-2 \hat{\mathbf{r}} \hat{\mathbf{r}})+\frac{\lambda}{c_{P}} \hat{\mathbf{n}} \hat{\mathbf{r}}+\frac{\mu}{c_{S}} \hat{\mathbf{r}} \hat{\mathbf{n}} .
$$

Symmetry of the impedance tensor is only obtained for normal incidence of the waves on the section considered, or for a special material parameter combination. The component form of the impedance tensor is given in Appendix A.

In the special case of normal incidence, $\hat{\mathbf{n}}=\hat{\mathbf{r}}$, the impedance tensor simplifies considerably:

$$
\mathbf{Z}=\frac{\lambda+2 \mu}{c_{P}} \hat{\mathbf{r}} \hat{\mathbf{r}}+\frac{\mu}{c_{S}}(\mathbf{1}-\hat{\mathbf{r}} \hat{\mathbf{r}}) .
$$

The wave speeds are given by, e.g., Achenbach [9], as

$$
c_{P}^{2}=\frac{\lambda+2 \mu}{\rho}, \quad c_{S}^{2}=\frac{\mu}{\rho}
$$

and impedance tensor (23) for normal incidence can then be written as

$$
\mathbf{Z}=\rho c_{P} \hat{\mathbf{r}} \hat{\mathbf{r}}+\rho c_{S}(\mathbf{1}-\hat{\mathbf{r}} \hat{\mathbf{r}})
$$

The first term is identified as a projection on the direction of propagation multiplied by the scalar impedance $\rho c_{P}$ of compression waves, and the second term is a projection on a transverse plane multiplied by the scalar impedance $\rho c_{S}$ of shear waves.

In the case of normal incidence, the impedance tensor is symmetric, while in the general case of oblique incidence it is not, except for a particular set of material parameters. The nature of the non-symmetry can be illustrated by rearranging general formula (22) in the following way:

$$
\begin{aligned}
\mathbf{Z}= & \frac{\lambda+2 \mu}{c_{P}}(\hat{\mathbf{n}} \cdot \hat{\mathbf{r}}) \hat{\mathbf{r}} \hat{\mathbf{r}}+\frac{\mu}{c_{S}}(\hat{\mathbf{n}} \cdot \hat{\mathbf{r}})(\mathbf{1}-\hat{\mathbf{r}} \hat{\mathbf{r}}) \\
& +\frac{\lambda}{c_{P}}[\hat{\mathbf{n}}-(\hat{\mathbf{n}} \cdot \hat{\mathbf{r}}) \hat{\mathbf{r}}] \hat{\mathbf{r}}+\frac{\mu}{c_{S}} \hat{\mathbf{r}}[\hat{\mathbf{n}}-(\hat{\mathbf{n}} \cdot \hat{\mathbf{r}}) \hat{\mathbf{r}}] .
\end{aligned}
$$

The terms in the first line are simply the normal incidence impedance multiplied by $(\hat{\mathbf{n}} \cdot \hat{\mathbf{r}})$. The vector in the bracket in the second line is the projection of the normal vector $\hat{\mathbf{n}}$ on the plane transverse to $\hat{\mathbf{r}}$ as illustrated in Figure 3. The magnitude of this vector is $\sin \psi$, where

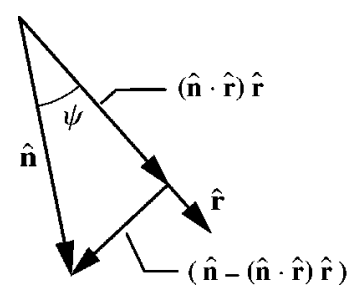

Figure 3. Identification of the transverse unit vector $\tilde{\mathbf{n}}$. 

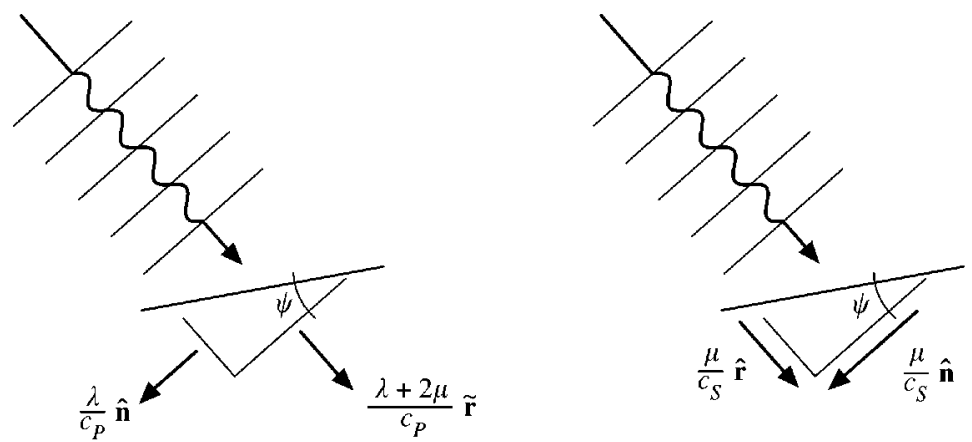

Figure 4. Stress contributions in plane P- and S-wave for oblique incidence.

$\psi$ denotes the angle between $\hat{\mathbf{n}}$ and $\hat{\mathbf{r}}$. When $\tilde{\mathbf{n}}$ denotes the corresponding transverse unit vector,

$$
\sin \psi \tilde{\mathbf{n}}=[\hat{\mathbf{n}}-(\hat{\mathbf{n}} \cdot \hat{\mathbf{r}}) \hat{\mathbf{r}}]
$$

and general impedance (26) can be written in the form

$$
\mathbf{Z}=\cos \psi\left[\frac{\lambda+2 \mu}{c_{P}} \hat{\mathbf{r}} \hat{\mathbf{r}}+\frac{\mu}{c_{S}}(\mathbf{1}-\hat{\mathbf{r}} \hat{\mathbf{r}})\right]+\sin \psi\left[\frac{\lambda}{c_{P}} \tilde{\mathbf{n}} \hat{\mathbf{r}}+\frac{\mu}{c_{S}} \hat{\mathbf{r}} \tilde{\mathbf{n}}\right] .
$$

The four terms in the impedance tensor for oblique incidence are the stress vectors acting on the triangle with side lengths $\cos \psi$ and $\sin \psi$ as illustrated in Figure 4 . The first two terms in equation (28) are the stress component on the wave front in $\mathrm{P}$ - and S-waves, respectively, while the last two terms are the stresses on a section along the direction of propagation, needed to provide the constraints inherent in $\mathrm{P}$ - and $\mathrm{S}$-waves respectively. Thus, impedance approximations for oblique incidence that neglect the constraint terms are inconsistent with the wave properties.

The general impedance tensor (26) is only symmetric, if the material parameters satisfy the condition

$$
\lambda / c_{P}=\mu / c_{S}
$$

Substitution of the wave speeds from equation (24) leads to the relations

$$
\lambda=2 \mu, \quad c_{P}=2 c_{S}
$$

where one relation follows from the other. This condition corresponds to a Poisson ratio of $v=\frac{1}{3}$. According to Achenbach [9], the wave speed ratio is $c_{P} / c_{S}=2 \cdot 00,2 \cdot 03,1 \cdot 85$ for copper, aluminum and steel, respectively, and thus the impedance tensor is nearly symmetric for these materials.

\section{SPHERICAL RADIATION FROM A POINT SOURCE}

Plane waves translate along the direction of propagation without change in magnitude, while waves radiating from a source of limited extent have curved wave fronts and decrease in magnitude with increased distance from the source. These features influence the radiation boundary condition and may impose restrictions on the distance of the radiating boundary from the source. 


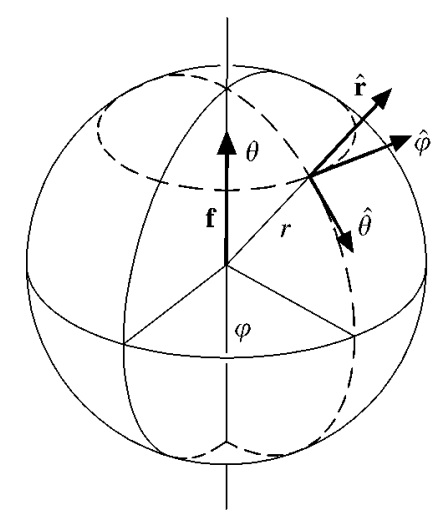

Figure 5. Time-dependent force $\mathbf{f}(t)$ in spherical $(r, \theta, \varphi)$ system.

An idealized case is the radiation of spherical waves from a point source. Due to the vector character, shear waves cannot have equal magnitude on a spherical wave front. In order to be specific, we therefore start the discussion from the wave field generated by point force $\mathbf{f}(t)$ acting at the center of a spherical co-ordinate $(r, \theta, \varphi)$ system with orthonormal coordinate vectors $\hat{\mathbf{r}}, \hat{\boldsymbol{\theta}}, \hat{\boldsymbol{\varphi}}$, shown in Figure 5.

The displacement field generated by a time-dependent force $\mathbf{f}(t)$ is given e.g., by Aki and Richards [10, chapter 4], in the following form:

$$
\begin{aligned}
\mathbf{u}= & \frac{1}{4 \pi \rho} \frac{1}{r^{3}}(3 \hat{\mathbf{r}} \hat{\mathbf{r}}-\mathbf{1}) \cdot \int_{r / c_{P}}^{r / c_{S}} \mathbf{f}(t-\tau) \tau \mathrm{d} \tau \\
& +\frac{1}{4 \pi \rho c_{P}^{2}} \frac{1}{r} \hat{\mathbf{r}} \hat{\mathbf{r}} \cdot \mathbf{f}\left(t-r / c_{P}\right)+\frac{1}{4 \pi \rho c_{S}^{2}} \frac{1}{r}(\mathbf{1}-\hat{\mathbf{r}} \hat{\mathbf{r}}) \cdot \mathbf{f}\left(t-r / c_{S}\right) .
\end{aligned}
$$

In the limit of large distance $r$, the integral term becomes small relative to the last two terms, given explicitly in terms of the force at a retarded time. These terms represent the farfield P-wave

$$
\mathbf{u}_{P}=\frac{1}{4 \pi \rho c_{P}^{2}} \frac{1}{r} \hat{\mathbf{r}} \hat{\mathbf{r}} \cdot \mathbf{f}\left(t-r / c_{P}\right)
$$

and the farfield S-wave

$$
\mathbf{u}_{S}=\frac{1}{4 \pi \rho c_{S}^{2}} \frac{1}{r}(\hat{\boldsymbol{\theta}} \hat{\boldsymbol{\theta}}+\hat{\boldsymbol{\varphi}} \hat{\boldsymbol{\varphi}}) \cdot \mathbf{f}\left(t-r / c_{S}\right),
$$

where $\hat{\mathbf{r}} \hat{\mathbf{r}}+\hat{\boldsymbol{\theta}} \hat{\boldsymbol{\theta}}+\hat{\boldsymbol{\varphi}} \hat{\boldsymbol{\varphi}}=\mathbf{1}$ has been used. The factor $r^{-1}$ describes the attenuation of the wave fields with distance from the source, due to conservation of energy.

In the case of spherical waves, the displacement fields $\mathbf{u}_{P}$ and $\mathbf{u}_{S}$ satisfy the identities

$$
\left(\frac{\partial}{\partial t}+\frac{c_{P}}{r} \frac{\partial}{\partial r} r\right) \mathbf{u}_{P}=\mathbf{0}
$$


and

$$
\left(\frac{\partial}{\partial t}+\frac{c_{S}}{r} \frac{\partial}{\partial r} r\right) \mathbf{u}_{S}=\mathbf{0}
$$

where the only difference from the plane wave identities is the inclusion of the co-ordinate $r$ inside the spatial differential operator.

\subsection{SPHERICAL P-WAVES}

The P-wave far field is a radial displacement of magnitude $u_{P}=\hat{\mathbf{r}} \cdot \mathbf{u}$. This displacement gives rise to the axial strain components

$$
\varepsilon_{r}=\frac{\partial u_{P}}{\partial r}, \quad \varepsilon_{\theta}=\varepsilon_{\varphi}=\frac{u_{P}}{r} .
$$

In addition, gradients along the wave front may produce shear strains, but in the present formulation variation along the wave front will be considered to be negligible, corresponding to a uniform spherical compression wave field or the situation at points of maximum intensity, e.g., in the directions $\pm \mathbf{f}$ in equation (32). The strain tensor can then be expressed as

$$
\boldsymbol{\varepsilon}_{P}=\hat{\mathbf{r}} \hat{\mathbf{r}} \varepsilon_{r}+\hat{\boldsymbol{\theta}} \hat{\boldsymbol{\theta}} \varepsilon_{\theta}+\hat{\boldsymbol{\varphi}} \hat{\boldsymbol{\varphi}} \varepsilon_{\varphi}=\hat{\mathbf{r}} \hat{\mathbf{r}}\left(\frac{1}{r} \frac{\partial\left(r u_{P}\right)}{\partial r}-\frac{2 u_{P}}{r}\right)+\mathbf{1} \frac{u_{P}}{r} .
$$

The spatial derivative can now be eliminated by use of the spherical P-wave identity (34), whereby

$$
\boldsymbol{\varepsilon}_{P}=-\hat{\mathbf{r}} \hat{\mathbf{r}}\left(\frac{1}{c_{P}} \frac{\partial u_{P}}{\partial t}+\frac{2 u_{P}}{r}\right)+\mathbf{1} \frac{u_{P}}{r} .
$$

The spherical P-wave field strain tensor consists of a radial part, of magnitude as defined by the parentheses, plus an isotropic part of magnitude $u_{P} / r$.

The stress vector $\tau$ on a section with normal $\hat{\mathbf{n}}$ can be obtained by a simple extension of the plane wave result (13), in which $\dot{\mathbf{u}}$ is replaced by $\dot{\mathbf{u}}+2 c_{P} r^{-1} \mathbf{u}$ and the contribution from the isotropic strain state is added:

$$
\tau_{P}=-\mathbf{Z}_{P} \cdot\left(\dot{\mathbf{u}}+\frac{2 c_{P}}{r} \mathbf{u}\right)+\frac{3 k}{r} \hat{\mathbf{n}} \hat{\mathbf{r}} \cdot \mathbf{u} .
$$

Upon substitution of the bulk modulus from equation (10) and the plane P-wave impedance tensor from equation (14) the following relation is obtained:

$$
\tau_{P}=-\mathbf{Z}_{P} \cdot \dot{\mathbf{u}}-\mathbf{R}_{P} \cdot \mathbf{u}
$$

with the plane $\mathrm{P}$-wave impedance tensor $\mathbf{Z}_{P}$ given by equation (14) and the spherical $\mathrm{P}$-wave radiation stiffness tensor

$$
\mathbf{R}_{P}=\frac{2 c_{P}}{r} \mathbf{Z}_{P}-\frac{3 k}{r} \hat{\mathbf{n}} \hat{\mathbf{r}}=\frac{1}{r}[4 \mu(\hat{\mathbf{n}} \cdot \hat{\mathbf{r}}) \hat{\mathbf{r}} \hat{\mathbf{r}}-(\lambda+2 \mu) \hat{\mathbf{n}} \hat{\mathbf{r}}] .
$$


It is observed that the spherical $\mathrm{P}$-wave radiation stiffness tensor $\mathbf{R}_{P}$ is not positive definite for all combinations of the material parameters $\lambda$ and $\mu$. A more detailed discussion of the radiation stiffness tensor for the full spherical wave field is given.

\subsection{SPHERICAL S-WAVES}

Consider shear wave field (32) and introduce the spherical co-ordinates such that the force points towards the pole, whereby $\hat{\boldsymbol{\theta}} \cdot \mathbf{f}=-\sin \theta$ and $\hat{\boldsymbol{\varphi}} \cdot \mathbf{f}=0$. The displacement field is then of the form $\mathbf{u}_{S}=\hat{\boldsymbol{\theta}} u_{S}$, where $u_{S}$ is proportional to $\sin \theta$. This displacement gives rise to the shear strain components

$$
2 \varepsilon_{r \theta}=2 \varepsilon_{\theta r}=\frac{\partial u_{S}}{\partial r}-\frac{2 u_{S}}{r},
$$

where the last term represents the effect of curvature of the wave front. The gradient along the spherical wave front gives rise to axial strain components, not associated with shear waves. These terms are neglected on the basis of the assumption that the variation along the wave front is small compared to the variation in the direction of propagation. Thus, the strain tensor associated with the spherical shear wave is

$$
\boldsymbol{\varepsilon}_{S}=\frac{1}{2}(\hat{\boldsymbol{\theta}} \hat{\mathbf{r}}+\hat{\mathbf{r}} \hat{\boldsymbol{\theta}})\left(\frac{1}{r} \frac{\partial\left(r u_{S}\right)}{\partial r}-\frac{2 u_{S}}{r}\right)=-\frac{1}{2}(\hat{\boldsymbol{\theta}} \hat{\mathbf{r}}+\hat{\mathbf{r}} \hat{\boldsymbol{\theta}})\left(\frac{1}{c_{S}} \frac{\partial u_{S}}{\partial t}+\frac{2 u_{S}}{r}\right),
$$

where the last equality follows from elimination of the spatial derivative by the spherical S-wave identity (35).

It is seen that a factor 2 appears in the last term, containing equal contributions from geometric wave attenuation $r^{-1}$ and curvature of the wave front. In acoustics, there is a general result that the area of a ray tube is proportional to the sum of the wave fronts two principal radii of curvature [11, p. 399], and thus the factor of two on the geometric attenuation term in equation (43) and the similar term in equation (38) for spherical waves appears to be a special instance of a more general relation.

The stress vector on a section with normal $\hat{\mathbf{n}}$ for a strain of form (43) without the last term has already been determined in the plane wave problem in section 2.2. The corresponding spherical S-wave solution follows immediately by including the full second factor from equation (42).

$$
\tau_{S}=-\mathbf{Z}_{S} \cdot\left(\dot{\mathbf{u}}+\frac{2 c_{S}}{r} \mathbf{u}\right)
$$

This is a relation of the form

$$
\tau_{S}=-\mathbf{Z}_{S} \cdot \dot{\mathbf{u}}-\mathbf{R}_{S} \cdot \mathbf{u}
$$

with the plane $\mathbf{S}$-wave impedance tensor $\mathbf{Z}_{S}$ given by equation (20) and the spherical S-wave radiation stiffness tensor

$$
\mathbf{R}_{S}=\frac{2 c_{S}}{r} \mathbf{Z}_{S}=\frac{2 \mu}{r}[\hat{\mathbf{r}} \hat{\mathbf{n}}+(\hat{\mathbf{n}} \cdot \hat{\mathbf{r}})(\mathbf{1}-2 \hat{\mathbf{r}} \hat{\mathbf{r}})]
$$

It is noted that the spherical radiation stiffness tensor for the shear wave is proportional to the shear wave impedance tensor, while the spherical radiation stiffness for compression waves contains an extra isotropic term. 


\subsection{THE SPHERICAL FARFIELD RADIATION CONDITION}

Under the condition that gradients along the wave front can be neglected, the stress vector generated by the compression and shear waves from a point source can be expressed in terms of the local total velocity and displacement vectors in the form

$$
\tau=-\mathbf{Z} \cdot \dot{\mathbf{u}}-\mathbf{R} \cdot \mathbf{u}
$$

where $\mathbf{Z}$ is the impedance tensor and $\mathbf{R}$ is an apparent stiffness tensor, generated by the spreading of the waves. The impedance tensor $\mathbf{Z}$ is identical to equation (22) corresponding to plane waves. The stiffness tensor $\mathbf{R}$ is given as the sum of the compression and shear wave contributions (41) and (46):

$$
\mathbf{R}=\mathbf{R}_{P}+\mathbf{R}_{S}=-\frac{\lambda+2 \mu}{r} \hat{\mathbf{n}} \hat{\mathbf{r}}+\frac{2 \mu}{r}[\hat{\mathbf{r}} \hat{\mathbf{n}}+(\hat{\mathbf{n}} \cdot \hat{\mathbf{r}}) \mathbf{1}]
$$

The stiffness decreases with distance from the source as $r^{-1}$ for a spherical source. The component form of the stiffness tensor is given in Appendix A.

The non-symmetry of the impedance tensor was already discussed in section 2.3. The discussion was facilitated by expressing the tensor in terms of the radial vector $\hat{\mathbf{r}}$ and the transverse unit vector $\tilde{\mathbf{n}}$. A representation of stiffness tensor (48) in a form similar to equation (28) is

$$
\mathbf{R}=\frac{\cos \psi}{r}[(2 \mu-\lambda) \hat{\mathbf{r}} \hat{\mathbf{r}}+2 \mu(\mathbf{1}-\hat{\mathbf{r}} \hat{\mathbf{r}})]-\frac{\sin \psi}{r}[(2 \mu+\lambda) \tilde{\mathbf{n}} \hat{\mathbf{r}}-2 \mu \hat{\mathbf{r}} \tilde{\mathbf{n}}] .
$$

It is seen that for oblique incidence, the stiffness tensor is non-symmetric for any values of the elastic parameters. The four terms may be interpreted as for the impedance tensor with reference to Figure 4. It is interesting to observe that the "axial stiffness" is $\cos \psi(2 \mu-\lambda) / r$, and therefore vanishes in the special case of $\lambda=2 \mu$, for which the general impedance tensor is symmetric. This corresponds to $c_{P}=2 c_{S}$, and thus the "axial stiffness" is seen to be positive for $c_{P}<2 c_{S}$ and negative for $c_{P}>2 c_{S}$.

\section{FREQUENCY RELATIONS AND THE NEAR FIELD}

The tensor boundary condition (47) is formulated in the time domain as a relation between the stress vector, the velocity vector and the displacement vector. However, in many problems it is of interest to analyze the response to a harmonic load. It is therefore of interest briefly to discuss the harmonic solution corresponding to the time-convolution formulation (31) for a concentrated force in an infinite medium. In a numerical solution, based on the approximate local radiation condition (47), the harmonic response will include the accumulated effect of repeated reflections from the fictitious boundary, caused by the approximate nature of local relation (47). Thus, the harmonic response is expected to constitute a severe test on the robustness of the local tensor boundary condition.

\subsection{HARMONIC TIME VARIATION}

Consider a point force $\mathbf{f}(t)$ with harmonic time variation with angular frequency $\omega$ and the displacement response $\mathbf{u}(t)$ of an infinite homogeneous elastic body. The harmonic time variation will be represented via the complex notation

$$
\mathbf{f}(t)=\overline{\mathbf{f}} \mathrm{e}^{-\mathrm{i} \omega t}, \quad \mathbf{u}(t)=\overline{\mathbf{u}} \mathrm{e}^{-\mathrm{i} \omega t} .
$$


The solution is expressed in terms of the wave numbers

$$
k_{P}=\omega / c_{P}, \quad k_{S}=\omega / c_{S} .
$$

It can be obtained either directly in the frequency domain, or by substituting force (50a) with harmonic time variation into equation (31). The result is, see e.g., reference [12, section 5.16],

$$
\overline{\mathbf{u}}=\frac{1}{4 \pi \rho} \frac{1}{r}\left\{(3 \hat{\mathbf{r}} \hat{\mathbf{r}}-\mathbf{1}) \frac{1}{\omega^{2}} \frac{\partial}{\partial r}\left[\frac{1}{r}\left(\mathrm{e}^{\mathrm{i} k_{P} r}-\mathrm{e}^{\mathrm{i} k_{s} r}\right)\right]+\frac{1}{c_{P}^{2}} \hat{\mathbf{r}} \hat{\mathbf{r}}^{\mathrm{i} k_{P} r}+\frac{1}{c_{S}^{2}}(\mathbf{1}-\hat{\mathbf{r}} \hat{\mathbf{r}} \mathrm{e})^{\mathrm{i} k_{s} r}\right\} \cdot \overline{\mathbf{f}} .
$$

The last two terms are identified as the farfield P- and S-wave, respectively, and it is easily verified that the first term decreases faster than $r^{-1}$, and thus becomes of decreasing importance with increasing distance.

The total power radiated by the P-and S-waves can be evaluated from the last two terms by integrating the power flux over a sphere,

$$
\begin{aligned}
& \left\langle\mathscr{P}_{P}\right\rangle=\int_{S} \frac{1}{2} c_{P} \rho \omega^{2} u_{P}^{2} \mathrm{~d} S=\frac{1}{3} \frac{1}{8 \pi} \frac{\omega^{2} f^{2}}{\rho c_{P}^{3}}, \\
& \left\langle\mathscr{P}_{S}\right\rangle=\int_{S} \frac{1}{2} c_{S} \rho \omega^{2} u_{S}^{2} \mathrm{~d} S=\frac{2}{3} \frac{1}{8 \pi} \frac{\omega^{2} f^{2}}{\rho c_{S}^{3}},
\end{aligned}
$$

where the factors $\frac{1}{3}$ and $\frac{2}{3}$ are due to the distribution of the P-and S-waves in the polar direction and around the equator respectively. It is seen that most of the energy is radiated by the S-wave.

\subsection{THE NEAR FIELD}

It is observed that $r \overline{\mathbf{u}}$ depends only on frequency and distance through the parameter $\omega r$, combining distance and frequency. Thus, the low-frequency domain may equally well be considered as the near field at any frequency. The near field is obtained by the expansion of equation (52) for small values of $\omega r$. The two-term expansion is

$$
\overline{\mathbf{u}} \simeq \frac{1}{4 \pi \rho} \frac{1}{r}\left[\frac{1}{c_{S}^{2}} \hat{\mathbf{r}} \hat{\mathbf{r}}+\frac{1}{2}\left(\frac{1}{c_{P}^{2}}+\frac{1}{c_{P}^{2}}\right)(\mathbf{1}-\hat{\mathbf{r}} \hat{\mathbf{r}})+\frac{\mathrm{i} \omega r}{3}\left(\frac{1}{c_{P}^{3}}+\frac{2}{c_{S}^{3}}\right) \mathbf{1}\right] \cdot \overline{\mathbf{f}} .
$$

This approximation is representative provided the second term is small relative to the first, i.e., provided $\omega r \ll c_{P, S}$.

It is notable that the imaginary part of $\overline{\mathbf{u}}$ is finite at $r=0$. In fact, the imaginary part at $r=0$ determines the velocity of the force, and thereby the power supplied by the force. The two power contributions (53), (54) in the far field can be recognized in the imaginary part of near field (53). Thus, while the imaginary part of the near field represents the power to be radiated through the far field, the real part represents the static solution. In the limit $\omega=0$, the near field reproduces Kelvin's static concentrated force solution

$$
\mathbf{u}=\frac{1}{8 \pi \mu} \frac{1}{r}\left[2 \hat{\mathbf{r}} \hat{\mathbf{r}}+\frac{\lambda+3 \mu}{\lambda+2 \mu}(\mathbf{1}-\hat{\mathbf{r}} \hat{\mathbf{r}})\right] \cdot \mathbf{f}
$$

as given by Love [13, p. 185]. 


\section{FINITE ELEMENT IMPLEMENTATION}

The practical implementation of the local tensor boundary condition in a finite-element-based procedure proceeds in the following steps. The formulation of the boundary condition is based on the assumption of linear elasticity, i.e., on the existence of a stress-strain relation of the form $\boldsymbol{\sigma}=\mathbf{D}: \boldsymbol{\varepsilon}$, where $\mathbf{D}$ is the elasticity tensor. When this relation and the local boundary condition (47) is substituted into weak formulation (2), the result is

$$
\begin{aligned}
& \int_{V} \tilde{\boldsymbol{\varepsilon}}: \mathbf{D}: \boldsymbol{\varepsilon} \mathrm{d} V+\int_{V} \rho \tilde{\mathbf{u}} \cdot \ddot{\mathbf{u}} \mathrm{d} V+\int_{S_{\infty}} \tilde{\mathbf{u}} \cdot \mathbf{Z} \cdot \dot{\mathbf{u}} \mathrm{d} S+\int_{S_{\infty}} \tilde{\mathbf{u}} \cdot \mathbf{R} \cdot \mathbf{u} \mathrm{d} S \\
& \quad=\int_{V} \tilde{\mathbf{u}} \cdot \mathbf{f} \mathrm{d} V+\int_{S_{\infty}} \tilde{\mathbf{u}} \cdot \tau \mathrm{d} V,
\end{aligned}
$$

where $S_{\infty}$ is the surface with the radiation boundary condition, while $S_{\tau}$ is the part of the boundary with prescribed stress vector $\tau$.

A typical finite element formulation is obtained by representing the actual and virtual displacement fields in the form

$$
\mathbf{u}(\mathbf{x}, t)=\mathbf{N}(\mathbf{x}) \mathbf{u}(t), \quad \tilde{\mathbf{u}}(\mathbf{x}, t)=\mathbf{N}(\mathbf{x}) \tilde{\mathbf{u}}(t),
$$

where $\mathbf{N}(\mathbf{x})$ are the spatial shape functions, and $\mathbf{u}(t)$ represents the time-dependent nodal displacement vector. The corresponding actual and virtual strain fields are

$$
\boldsymbol{\varepsilon}(\mathbf{x}, t)=\mathbf{B}(\mathbf{x}) \mathbf{u}(t), \quad \tilde{\boldsymbol{\varepsilon}}(\mathbf{x}, t)=\mathbf{B}(\mathbf{x}) \tilde{\mathbf{u}}(t),
$$

where the strain shape functions $\mathbf{B}(\mathbf{x})$ are obtained from the displacement shape functions $\mathrm{N}(\mathbf{x})$ by differentiation.

The substitution of representations (58), (59) into equation (57) leads to a system of ordinary second order differential equations of the form

$$
M \ddot{u}+C \dot{u}+K u=f
$$

with mass matrix

$$
\mathrm{M}=\int_{V} \rho \mathrm{N}^{\mathrm{T}} \mathrm{N} \mathrm{d} V
$$

damping matrix

$$
\mathrm{C}=\int_{S_{\infty}} \mathrm{N}^{\mathrm{T}} \mathbf{Z ~ N ~ d S}
$$

stiffness matrix

$$
\mathrm{K}=\int_{V} \mathrm{~B}^{\mathrm{T}} \mathbf{D} \mathrm{B} \mathrm{d} V+\int_{S_{\infty}} \mathrm{N}^{\mathrm{T}} \mathbf{R} \mathbf{N} \mathrm{d} S
$$

and load vector

$$
\mathbf{f}=\int_{V} \mathrm{~N}^{\mathrm{T}} \mathbf{f} \mathrm{d} V+\int_{S_{\tau}} \mathrm{N}^{\mathrm{T}} \tau \mathrm{d} S .
$$


It is seen that the impedance term in the boundary conditions generates a damping matrix, while the apparent boundary spring stiffness generates an extra term in the traditional volume-based stiffness matrix.

\section{EXAMPLES}

The performance of the local tensor radiation boundary condition is illustrated by two examples. The first example is the generation of a wave field by a time-dependent concentrated force in an infinite elastic medium. In this problem, the only natural length scale is the wavelength(s) associated with the the wave field. The quality of the tensor boundary condition increases with the distance of the radiation boundary from the source, while the purpose of radiating boundary conditions is to limit the extent of the computational domain. The example illustrates the quality of the solution for a radiation boundary as close as $0.7 \mathrm{P}$-wave length or little more than a full $\mathrm{S}$-wave length. It is an important feature of the local tensor radiation boundary condition that it is formulated in the time domain, and the example therefore illustrates the radiation of $\mathrm{P}$ - and $\mathrm{S}$-waves from a time-limited pulse. A frequency analysis is then used to demonstrate that even for a rather close radiation boundary, there are only very small accumulated effects of boundary reflections. In this first example, analytical solutions are available both in the time and the frequency domains, and the accuracy of the numerical solution can be demonstrated in absolute terms. In practice, radiation boundary conditions are to be used in problems where no analytical solution is available, often in connection with a non-trivial local geometry. This is illustrated in the second example, providing a time domain analysis of a pulse load on the floor of an underground gallery.

The purpose of the time domain analysis is to investigate the ability of the local tensor boundary condition to let $\mathrm{P}$ - and S-waves pass without reflections or conversion between wave types. This is best accomplished by selecting a pulse with a well-defined duration in order to enable the identification of arrival times of direct and reflected waves.

The pulse shape was selected from the polynomial family defined by

$$
f(t)=\tau\left(1-\tau^{2}\right)^{n}, \quad-1<\tau<1
$$

with $\tau=2 t / T-1$. The total duration of the pulse is $T$, and the integer $n$ leads to vanishing derivatives of order $0,1, n-1$ at the ends of the pulse. The frequency content of the pulse is determined from the Fourier transform

$$
F(\omega)=\int_{V}^{T} f(t) \mathrm{e}^{\mathrm{i} \omega t} \mathrm{~d} t
$$

Using the integral representations of Bessel functions, given e.g., by Abramowitz and Stegun [14], the following explicit result is found,

$$
F(\boldsymbol{\Omega})=\mathrm{i} T \mathrm{e}^{\mathrm{i} \boldsymbol{\Omega}} \frac{n !}{(\boldsymbol{\Omega} / 2)^{n}} j_{n+1}(\boldsymbol{\Omega}), \quad \boldsymbol{\Omega}=\frac{1}{2} \omega T,
$$

where $\boldsymbol{\Omega}$ is a non-dimensional frequency corresponding to the pulse duration $T$, and $j_{n}()$ is the spherical Bessel function of the first kind of order $n$. The complex exponential factor arises because the pulse is shifted to start at time $t=0$.

The present analyses are based on $n=2$, leading to a continuous slope at the ends of the pulse. This pulse is shown in Figure 6(a), and the corresponding spectral density function is 

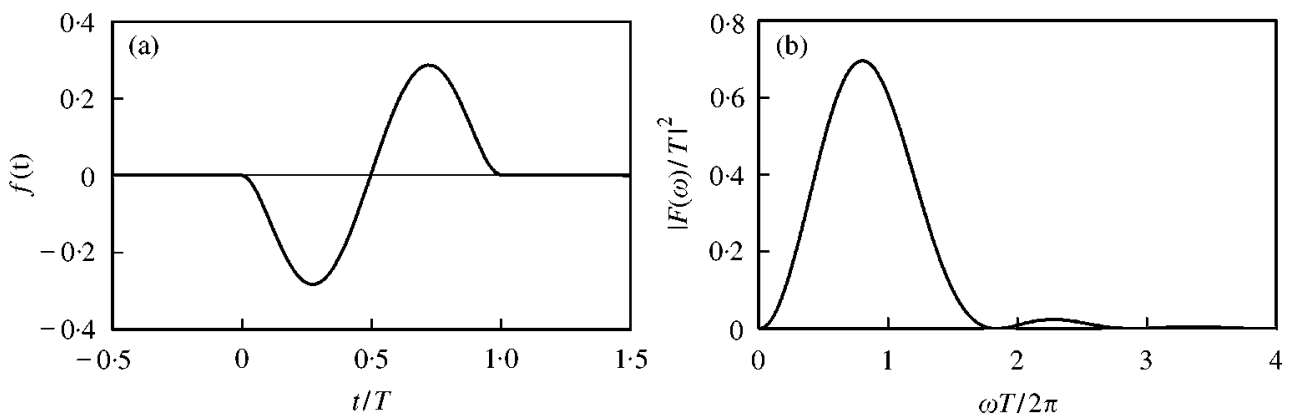

Figure 6. Force pulse, $n=2$ : (a) time history; (b) spectral density.

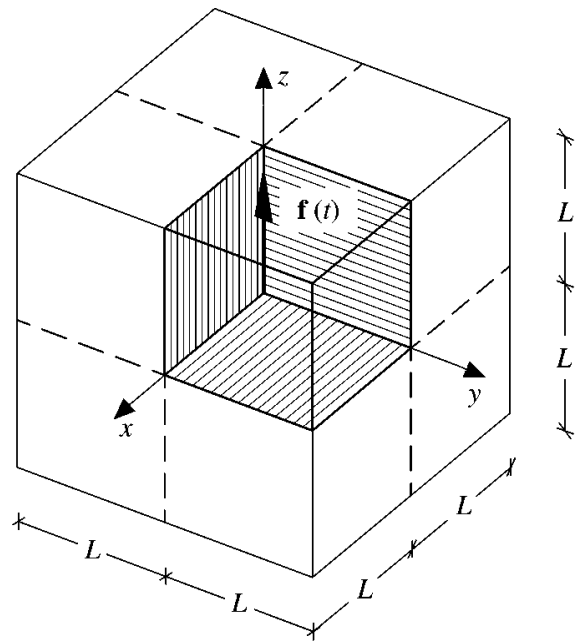

Figure 7. Elastic cube with side length $2 L$, and the octant used in the finite element model.

shown in Figure 6(b). It is seen that the frequency content is centered around $0 \cdot 8 / T$ with very small side lobes. However, it should be noted that an accurate representation of the response requires an accurate representation of frequency components up to around $1 \cdot 5 / T$, imposing requirements on the necessary number of elements per wavelength.

\subsection{CONCENTRATED FORCE IN 3-D}

The local tensor boundary condition (47) has been tested in the time and frequency domains by the concentrated force problem illustrated in Figure 7. Ideally, the force $\mathbf{f}(t)$ located at the origin of the co-ordinate system is assumed to act in an infinite isotropic elastic continuum. The model consists of a finite element discretization of the first octant with side length $L=25 \mathrm{~m}$ using simple 8-node elements with linear displacement interpolation and side length $h=1 \mathrm{~m}$. In both types of analyses, the material was specified with wave speeds $c_{P}=20 \mathrm{~m} / \mathrm{s}$ and $c_{S}=10 \mathrm{~m} / \mathrm{s}$, corresponding to the special case of symmetric impedance matrix. The mass density is $\rho=2000 \mathrm{~kg} / \mathrm{m}^{3}$, and the maximum value of the force is $f_{\max }=1 \mathrm{MN}$. 

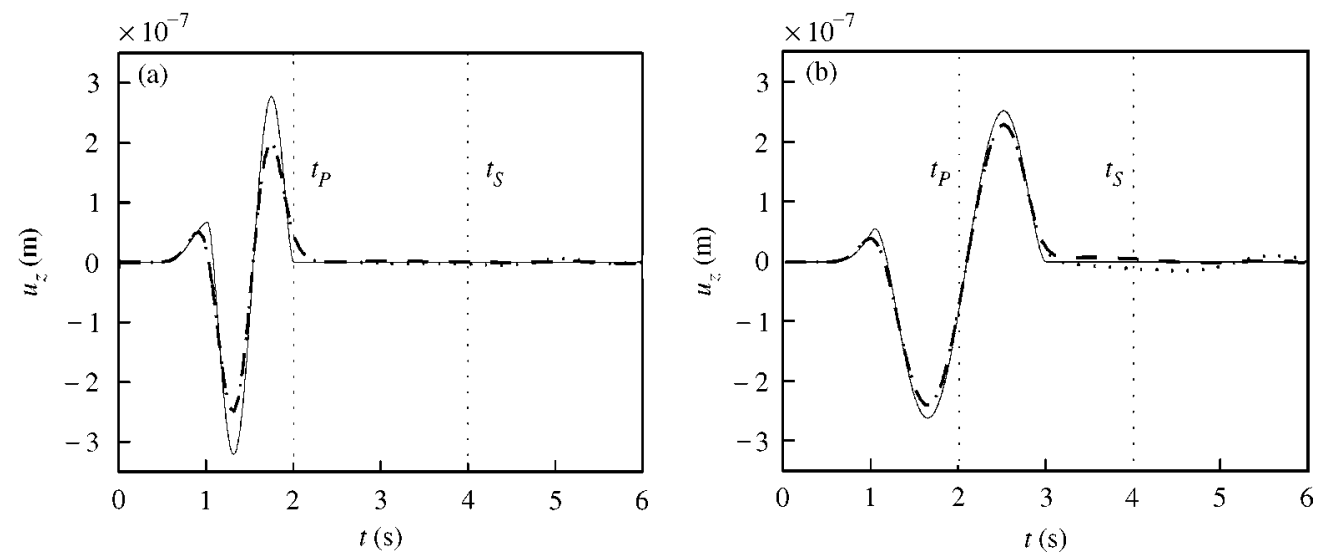

Figure 8. Displacement component $u_{z}$ at $(x, y, z)=(10,0,0)$ : (a) $T=1 \mathrm{~s}$; (b) $T=2 \mathrm{~s}$. Analytical: - , FEM $(\mathrm{Z}+\mathrm{R}):----$, FEM $(\mathrm{Z}): \cdots \cdots \cdot$.
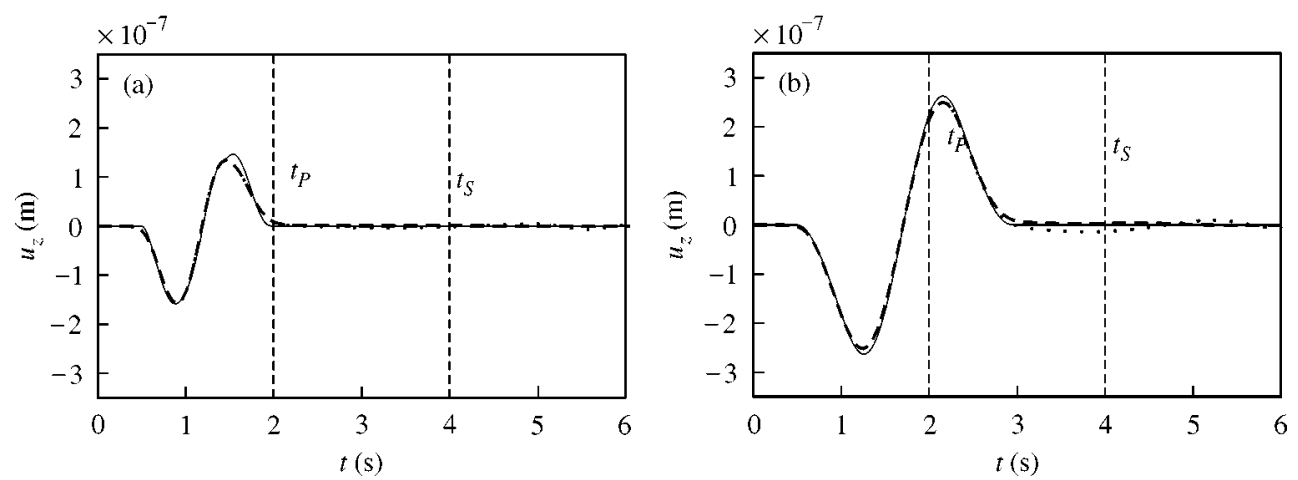

Figure 9. Displacement component $u_{z}$ at $(x, y, z)=(0,0,10):$ (a)) $T=1 \mathrm{~s} ;$ (b) $T=2 \mathrm{~s}$. Analytical: - , FEM $(\mathrm{Z}+\mathrm{R})$ : - - - , FEM $(\mathrm{Z}): \cdots \cdots \cdot$

\subsubsection{Time domain analysis}

The results in Figures 8 and 9 show the time history of the displacement component $u_{z}$ at three different locations for impedance and combined impedance and stiffness radiation boundary conditions, and for two different pulse duration times, $T=1$ and $2 \mathrm{~s}$. With a pulse duration of $T=1 \mathrm{~s}$, the dominating $\mathrm{P}$-wavelength is about $c_{P} T / 0 \cdot 8=25 \mathrm{~m}$, i.e., equal to the distance $L$ from the center to the sides of the cube. Thus, the radiation boundary condition is imposed fairly close to the source. For a pulse duration of $T=2 \mathrm{~s}$, the distance from the source to the sides is only half of the dominating P-wavelength.

Figure 8 shows the time histories at the point $(x, y, z)=(10,0,0)$, a displacement generated by shear waves in the transverse plane. For the pulse duration $T=1 \mathrm{~s}$, the dominating S-wavelength is around $12.5 \mathrm{~m}$. The higher frequency components are therefore represented by less than 10 elements per wavelength. As a consequence, the ability of the element mesh to transmit the pulse shape of the shear wave is limited as illustrated in Figure 8 (a). For pulse duration $T=2 \mathrm{~s}$, the element density per wavelength is double, and the representation of the pulse shape is much improved as shown in Figure 8(b). The figures show the analytical results together with the impedance radiation condition as a dotted line, and the combined impedance and stiffness condition as a dash-dot line. In the case of the 

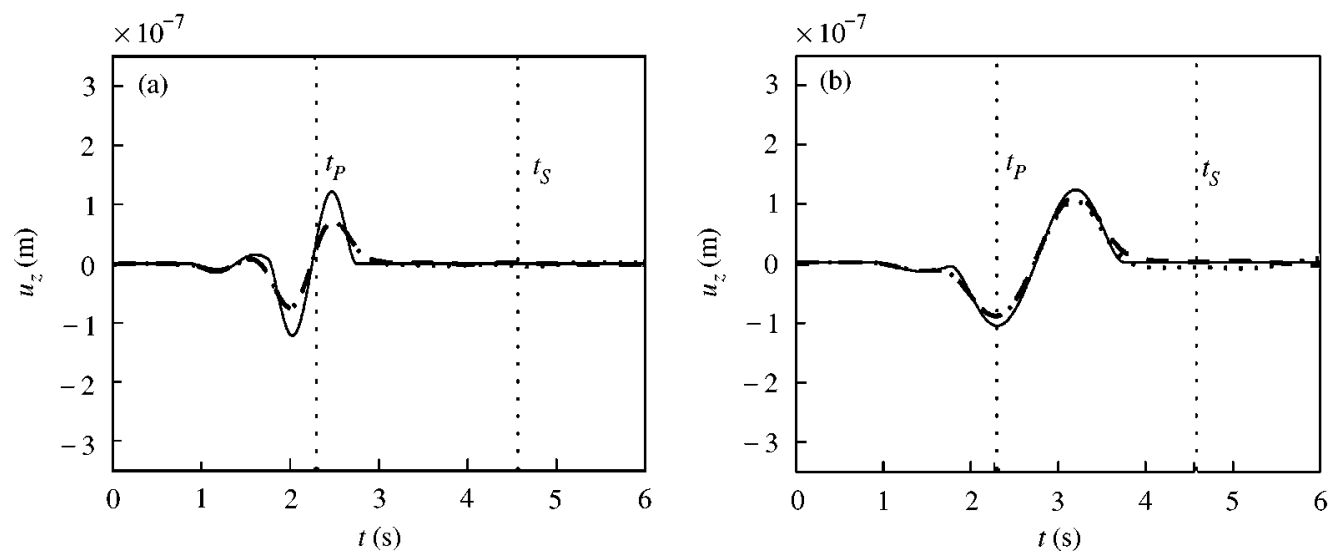

Figure 10. Displacement component $u_{z}$ at $(x, y, z)=(10,10,10)$ : (a) $T=1 \mathrm{~s}$; (b) $T=2 \mathrm{~s}$. Analytical: - , FEM $(\mathrm{Z}+\mathrm{R}):----$, FEM $(\mathrm{Z}): \cdots \cdots$.

(a)

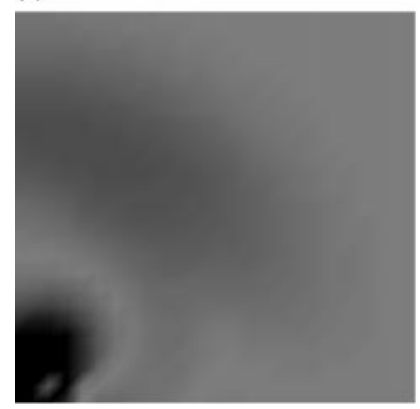

(b)

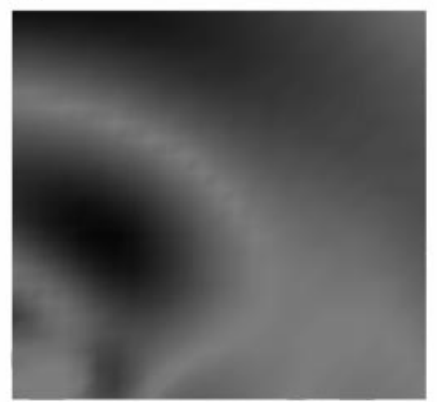

(c)

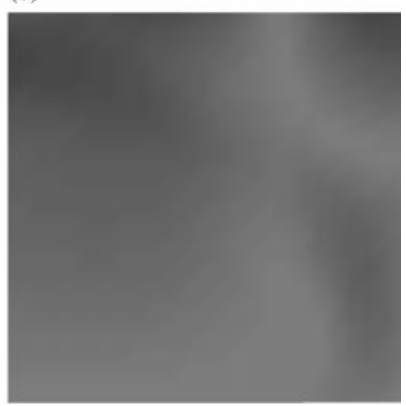

Figure 11. P-wave strain energy density in $x z$ plane: (a) $t=1.25$; (b) 2.5 and (c) $4.0 \mathrm{~s}$.

short pulse the distance to the boundary, measured as a full P-wavelength, is sufficiently large for both formulations to be accurate, while for the longer pulse with a distance to the boundary of only half the P-wavelength, the combined impedance and stiffness condition is superior. The times $t_{P}$ and $t_{S}$ in the figure indicate the arrival of reflected $\mathrm{P}$ - and $\mathrm{S}$-waves, respectively, from the closest point of the boundary. In this case, there is no direct reflection of $\mathrm{P}$-waves from the closest point although there is a small precursor generated by $\mathrm{P}$-waves at small angle, but even the S-waves do not show any distinct reflection from the approximate radiation conditions.

Figures 9 and 10 show the similar results for a point $(x, y, z)=(0,0,10)$ ahead of the force and a point $(x, y, z)=(10,10,10)$ on the diagonal of the cube. The pulse shape is better represented by the finite element results for the point ahead of the force shown in Figure 9, because the displacement at this point is due to $\mathrm{P}$-waves with a longer wavelength than the $\mathrm{S}$-waves dominating the results of Figure 8. Apart from this, the conclusion to be drawn from the results are the same as before, namely that the combined impedance stiffness radiation boundary condition remains accurate, even for boundaries only half a P-wavelength from the source.

The progress of the waves through the cube for a pulse duration of $T=2 \mathrm{~s}$ is further illustrated in Figures 11 and 12, showing the strain energy density of the P-waves and the S-waves, respectively, at times $t=1 \cdot 25,2 \cdot 5,4 \cdot 0 \mathrm{~s}$. Figures 11(a) and 11(b) clearly show the angular variation of the $\mathrm{P}$-wave field, while the P-wave has nearly left the cube in 
(a)

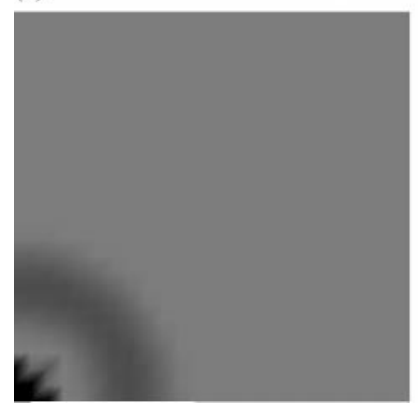

(b)

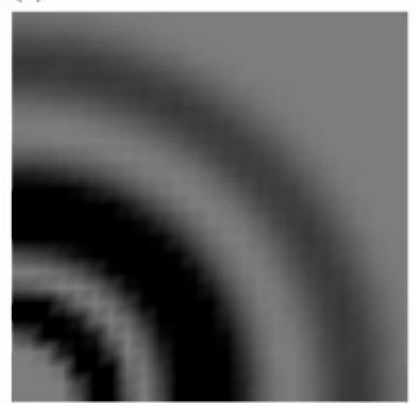

(c)

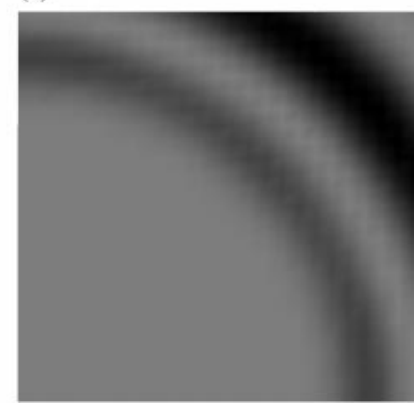

Figure 12. S-wave strain energy density in $x y$ plane: (a) $t=1.25$; (b) $2.5 \mathrm{~s}$ and (c) $4.0 \mathrm{~s}$.
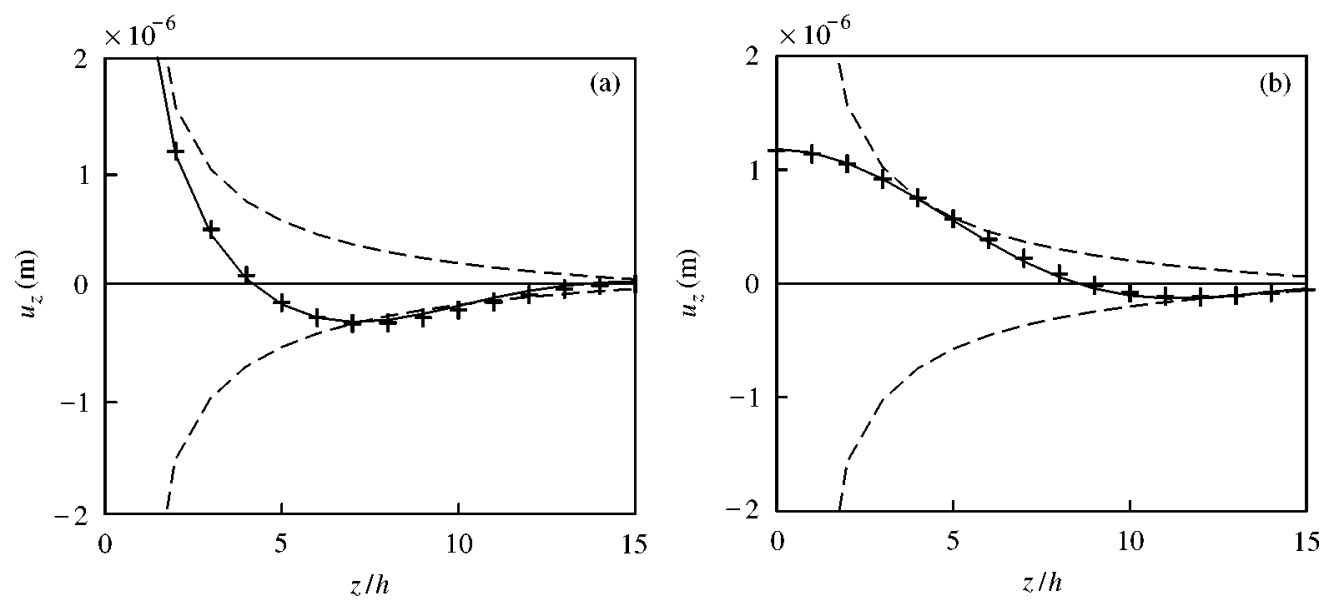

Figure 13. Displacement component $u_{z}$ along $z$-axis: (a) $\operatorname{Re}\left[u_{z}\right]$; (b) $\operatorname{Im}\left[u_{z}\right]$. Analytical: $-, \operatorname{FEM}(\mathrm{Z}+\mathrm{R}):+$.

Figure 11(c). The circular wave front pattern of the S-waves in the transverse plane is illustrated in Figure 12, where Figure 12(c) shows the undisturbed passage of the radiating boundary without visible reflections.

\subsubsection{Frequency domain analysis}

While a time domain analysis gives an impression of the ability to conserve pulse shape and wave fronts, a frequency domain analysis can illustrate the accumulated effect of spurious reflections from the approximate radiation boundary condition. The frequency domain problem requires solution of the full set of complex equations, and therefore the octant in the frequency model consists of only $15 \times 15 \times 15$ elements with side length $h=1 \mathrm{~m}$.

Figures 13(a) and 13(b) show the real and imaginary part of the displacement component $u_{z}$ along the $z$-axis for a concentrated force with harmonic time variation with period $T=1 \cdot 2 \mathrm{~s}$, calculated using the combined impedance stiffness radiation boundary condition. The agreement with the analytical solution is seen to be excellent, indicating negligible effect of spurious reflections. Similar agreement is found for the displacement component $u_{z}$ along the $x$-axis, shown in Figure 14.

It is remarkable, that although the element shape functions fail to capture the innermost two points of the singular real part, the rest of the points agree very well with the analytical 

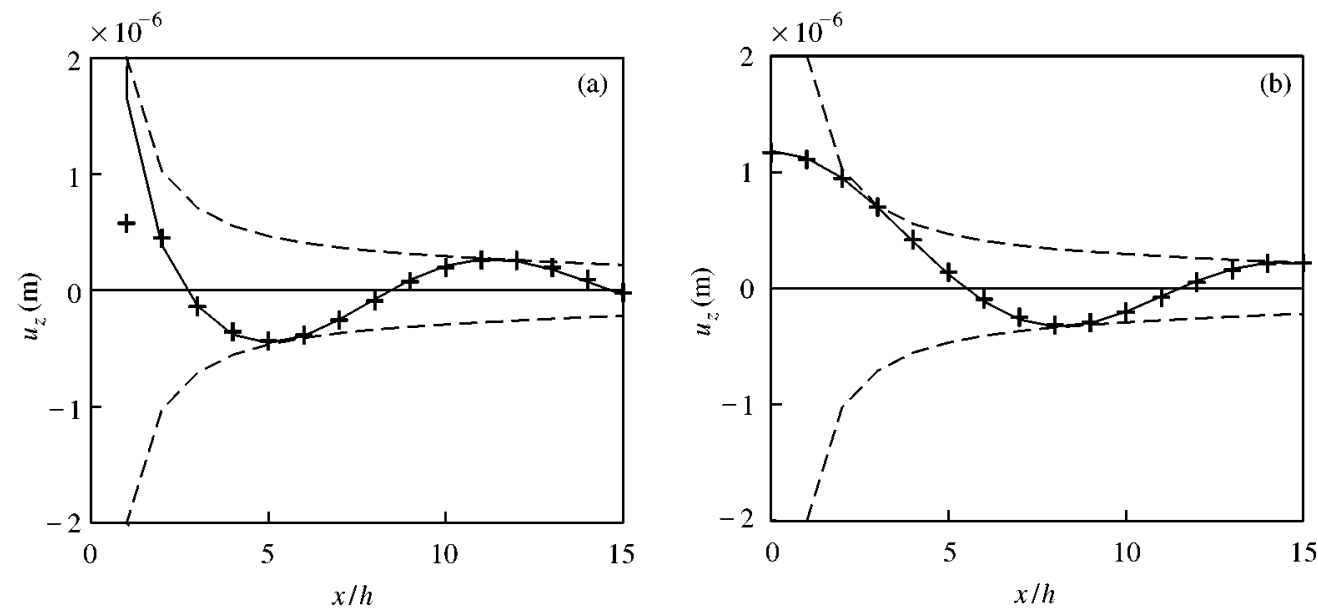

Figure 14. Displacement component $u_{z}$ along $x$-axis: (a) $\operatorname{Re}\left[u_{z}\right]$; (b) $\operatorname{Im}\left[u_{z}\right]$. Analytical: $\longrightarrow, \operatorname{FEM}(\mathrm{Z}+\mathrm{R}):+$.
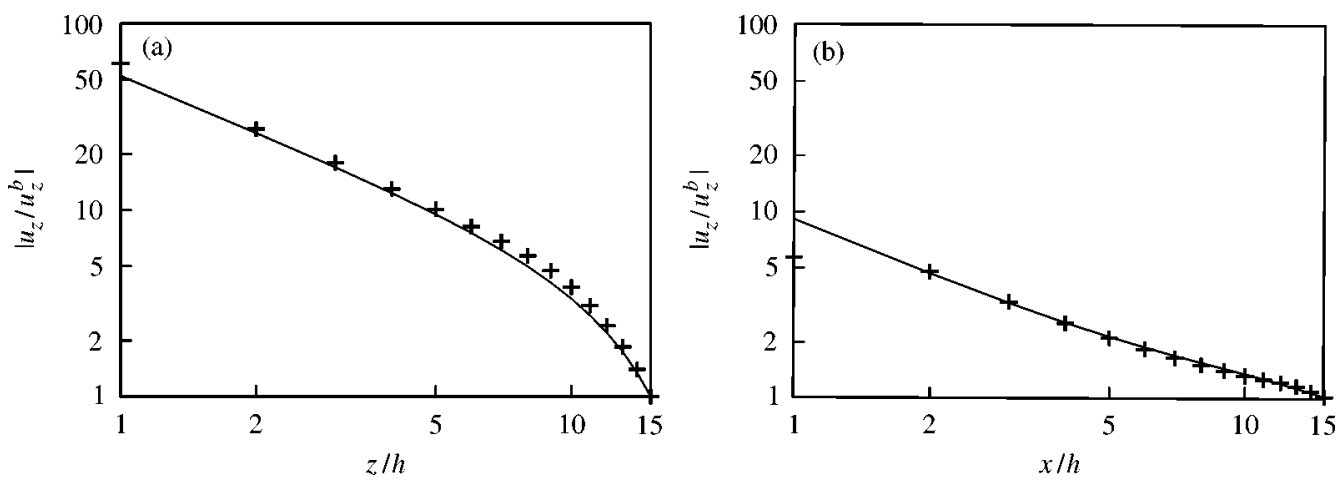

Figure 15. Displacement component $\left|u_{z}\right|$ normalized with value $\left|u_{z}^{b}\right|$ at the boundary: (a) $z$-axis; (b) along $x$-axis. Analytical: —, FEM $(\mathrm{Z}+\mathrm{R}):+$.

solution. The degree of accuracy is illustrated in Figure 15, showing the amplitude on a logarithmic scale along the $z$-and the $x$-axis. Again, the agreement is seen to be good, and no noticeable effect of reflections is seen.

\subsection{WAVES FROM UNDERGROUND GALLERY}

An important application of the tensor radiation boundary condition is the design for the dynamic effects of underground structures. An example is the underground gallery shown in Figures 16 and 17. The total width and height of the gallery are $L$, with only the right half shown due to symmetry. The computational domain extends $L$ below, above, and to the side of the gallery. The domain is discretized using bilinear elastic elements with 24 elements per length $L$. The Poisson ratio is $v=0 \cdot 3$, corresponding to $c_{S} / c_{P}=0 \cdot 55$. For this material, the impedance and radiation stiffness matrices are non-symmetric.

The loading is a vertical force at the center of the floor of the gallery with a time variation described by the pulse shape (65) with $n=2$ shown in Figure 6(a). The time period is chosen 
(a)

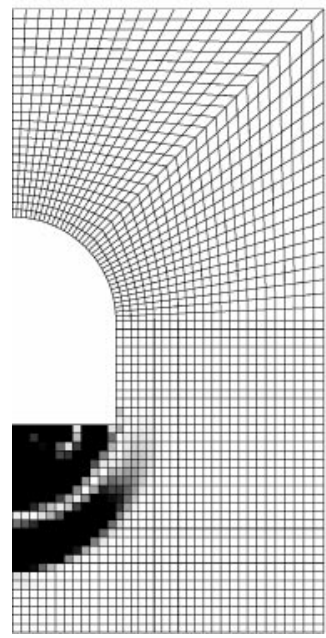

(b)

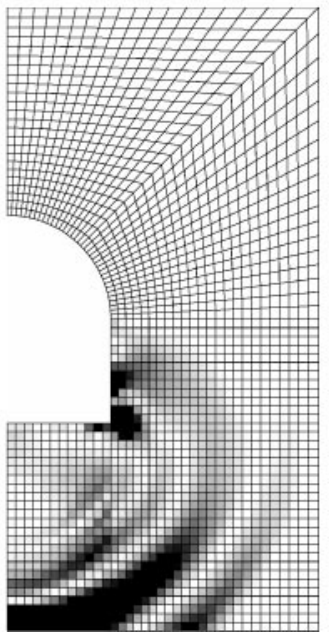

(c)

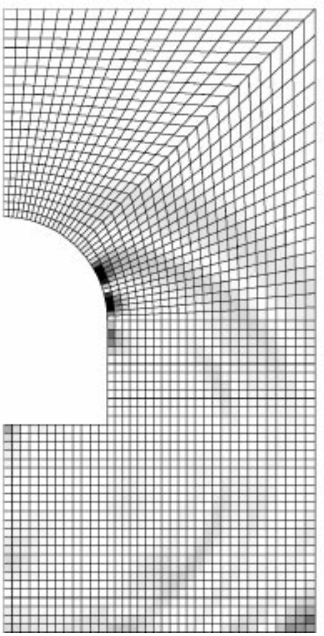

(d)

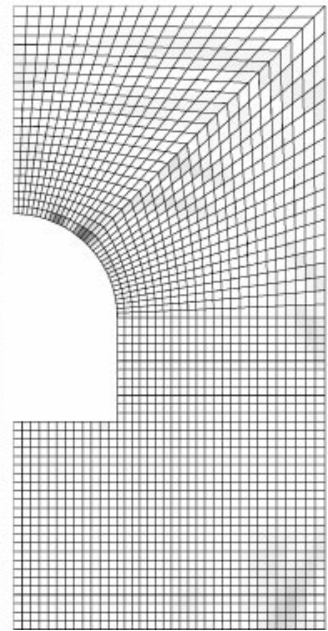

Figure 16. P-wave strain energy density: (a) $t=T$; (b) $2 T$; (c) $3 T$ and (d) $4 T$.

(a)

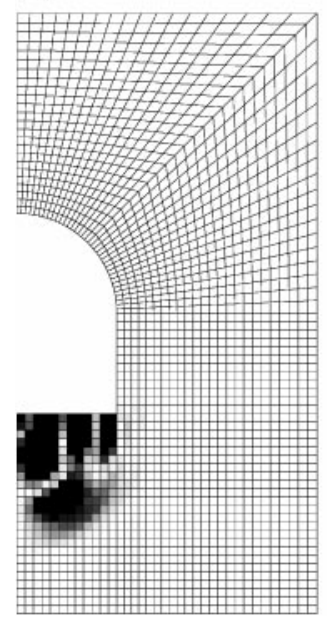

(b)

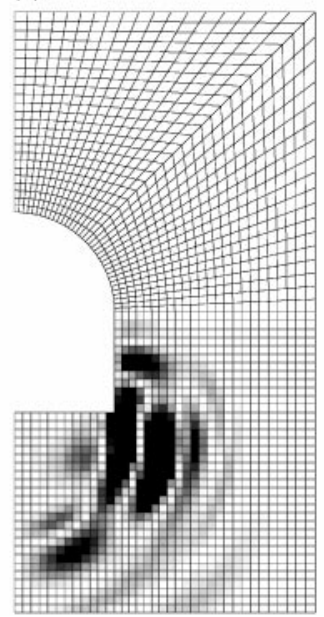

(c)

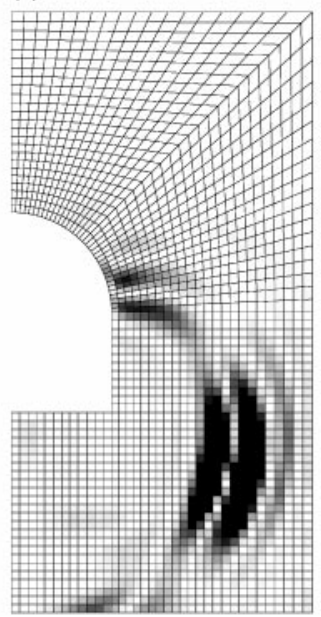

(d)

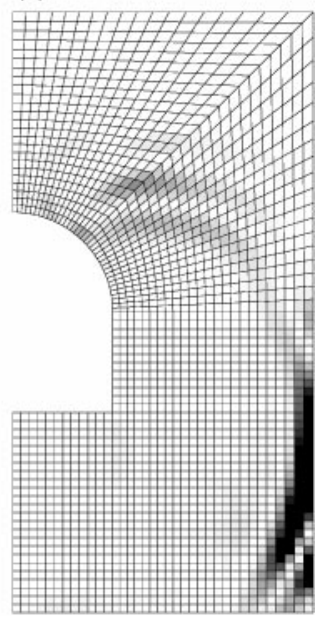

Figure 17. S-wave strain energy density: (a) $t=T$; (b) $2 T$; (c) $3 T$ and (d) $4 T$.

such that $c_{P} T=L$. Thus, the full $\mathrm{P}$-wave fits precisely between the gallery floor and the lower boundary of the computational domain. The time $t=0$ denotes the start of the pulse, and thus the times $t=T, 2 T, 3 T, 4 T$ used in Figures 16 and 17 correspond to 1, 2, 3 and 4 times the time $T$ needed for the P-wave to reach the lower boundary of the computational domain. The strain energy density of the P-wave is shown in Figure 16. The plots have been scaled to the range of Figure 16(c) whereby the first two plots are shown with extra contrast. The last two plots show a P-wave folding around the gallery and demonstrate the absence of reflections of the P-wave from the computational boundary. Figure 17 shows similar results for the S-wave energy density. The S-waves spread nearly horizontally, and the last two plots demonstrate the absence of reflections from the computational boundary. 


\section{DISCUSSION}

Local radiation boundary conditions have been formulated in the form of an impedance tensor and a stiffness tensor, linking the stress vector at the radiating boundary to the velocity and the displacement vectors. While the impedance tensor represents a propagating plane wave, the effect of spreading of the wave is accounted for via an elastic stiffness tensor. For non-normal wave incidence, the impedance and stiffness tensors are non-symmetric, and the origin of the non-symmetry is explained via the need for transverse stresses in propagating elastic waves. The basic assumptions of the local boundary condition is that the general direction of wave propagation is known or estimated, and that gradients along the wave front can be neglected. The latter assumption is tested by considering radiation from a concentrated force, and it is demonstrated by finite element time and frequency domain calculations that gradient effects are negligible, even for radiation boundaries placed as close as half a P-wavelength from the source. In a recent paper, a generalization of the local tensor boundary condition to moving loads was demonstrated to work satisfactorily [15].

The boundary conditions of the present paper are local in the sense that the stress vector at a point of an internal radiation boundary is expressed in terms of the displacement and velocity vectors at that particular point. Clearly, the accuracy of this approximation depends on the dominating direction of propagation of the wave field to be radiated. The concept can be generalized by eliminating the explicit dependence on the dominating propagation direction. In a recent paper on the scalar wave equation, the elimination of the propagation direction was accomplished via introduction of suitable differential operators in the plane of the radiating surface, and the relation to previous higher order boundary condition was established [7]. The method leads directly from the field equations to a set of "internal variables" in the radiating surface, and thus relates to the work of Paronesso and Wolf [5], in which internal variables were introduced on the basis of the discretized global equations. An extension of the direct field equation approach to elastic wave propagation is in progress.

\section{ACKNOWLEDGMENTS}

This work has been supported by the Danish Technical Research Council through the project Damping Mechanisms in Dynamics of Structures and Materials.

\section{REFERENCES}

1. J. LySMER and R. L. KUHLEMEYER 1969 Journal of the Engineering Mechanics Division, American Society of Civil Engineers 95, 859-877. Finite dynamic model for infinite media.

2. J. P. WOLF 1986 Earthquake Engineering and Structural Dynamics 14, 655-673. A comparison of time-domain transmitting boundaries.

3. L. X. KelleZI 1998 Ph.D. Thesis, Department of Structural Engineering and Materials, Technical University of Denmark. Dynamic soil-structure-interaction, Transmitting boundary for transient analysis.

4. J. P. Wolf and C. Song 1996 Finite Element Modelling of Unbounded Media. New York: Wiley.

5. A. PARONESSO and J. P. WOLF 1998 Earthquake Engineering and Structural Dynamics 27, 609-618. Recursive evaluation of interaction forces and property matrices from unit-impulse response functions of unbounded medium based on balancing approximation.

6. D. Givoli 1991 Journal of Computational Physics 94, 1-29. Non-reflecting boundary conditions.

7. S. KRENK 2001 International Journal for Numerical Methods in Engineering. Unified formulation of radiation conditions for the wave equation (in press). 
8. D. Givoli 1999 Applied Mechanics Review 52, 333-349. Exact representations on artificial interfaces and applications in mechanics.

9. J. D. ACHENBACH 1973 Wave Propagation in Elastic Solids. Amsterdam: North-Holland.

10. K. AKI and P. G. Richards 1980 Quantitative Seismology, Vol. 1. New York: Freeman.

11. A. D. PIERCE 1981 Acoustics: An Introduction to its Physical Principles and Applications. New York: McGraw-Hill.

12. A. C. ERingen and E. S. Suhubi 1975 Elastodynamics, Vol. II, Linear Theory. New York: Academic Press.

13. A. E. H. Love 1944 A Treatise on the Mathematical Theory of Elasticity. New York: Dover Publications: fourth edition.

14. M. Abramowitz and I. Stegun 1968 Handbook of Mathematical Functions. New York: Dover Publications.

15. S. Krenk, L. Kellezi, S. R. K. Nielsen and P. H. KirkegaArd 1999 Structural Dynamics, Eurodyn '99, Vol. 1, 447-452. Rotterdam: Balkema. Finite elements and transmitting boundary conditions for moving loads.

\section{APPENDIX A: MATRIX NOTATION}

The derivations and results of the main paper have been given in the notation of vector analysis. In this notation, the scalar product of the unit vectors $\hat{\mathbf{n}}$ and $\hat{\mathbf{r}}$ is written as $\hat{\mathbf{n}} \cdot \hat{\mathbf{r}}$, while the vector dyad $\hat{\mathbf{n}} \hat{\mathbf{r}}$ without the dot represents an exterior product. In terms of the Cartesian vector components $\hat{\mathbf{n}}=\left(\hat{n}_{1}, \hat{n}_{2}, \hat{n}_{3}\right)$ and $\hat{\mathbf{r}}=\left(\hat{r}_{1}, \hat{r}_{2}, \hat{r}_{3}\right)$, the corresponding components of the vector dyad takes the form of a matrix,

$$
[\hat{\mathbf{n}} \hat{\mathbf{r}}]=\left[\begin{array}{lll}
\hat{n}_{1} \hat{r}_{1} & \hat{n}_{1} \hat{r}_{2} & \hat{n}_{1} \hat{r}_{3} \\
\hat{n}_{2} \hat{r}_{1} & \hat{n}_{2} \hat{r}_{2} & \hat{n}_{2} \hat{r}_{3} \\
\hat{n}_{3} \hat{r}_{1} & \hat{n}_{3} \hat{r}_{2} & \hat{n}_{3} \hat{r}_{3}
\end{array}\right] .
$$

The main results of the paper are the impedance tensor $\mathbf{Z}$ in equation (22) with component form

$$
\begin{aligned}
& {\left[Z_{i j}\right]=\frac{2 \mu}{c_{P}}(\hat{\mathbf{n}} \cdot \hat{\mathbf{r}})\left[\begin{array}{lll}
1 & 0 & 0 \\
0 & 1 & 0 \\
0 & 0 & 1
\end{array}\right]-2 \mu\left(\frac{1}{c_{S}}-\frac{1}{c_{P}}\right)(\hat{\mathbf{n}} \cdot \hat{\mathbf{r}})\left[\begin{array}{lll}
\hat{r}_{1} \hat{r}_{1} & \hat{r}_{1} \hat{r}_{2} & \hat{r}_{1} \hat{r}_{3} \\
\hat{r}_{2} \hat{r}_{1} & \hat{r}_{2} \hat{r}_{2} & \hat{r}_{2} \hat{r}_{3} \\
\hat{r}_{3} \hat{r}_{1} & \hat{r}_{3} \hat{r}_{2} & \hat{r}_{3} \hat{r}_{3}
\end{array}\right]} \\
& +\frac{\lambda}{c_{P}}\left[\begin{array}{lll}
\hat{n}_{1} \hat{r}_{1} & \hat{n}_{1} \hat{r}_{2} & \hat{n}_{1} \hat{r}_{3} \\
\hat{n}_{2} \hat{r}_{1} & \hat{n}_{2} \hat{r}_{2} & \hat{n}_{2} \hat{r}_{3} \\
\hat{n}_{3} \hat{r}_{1} & \hat{n}_{3} \hat{r}_{2} & \hat{n}_{3} \hat{r}_{3}
\end{array}\right]+\frac{\mu}{c_{S}}\left[\begin{array}{lll}
\hat{r}_{1} \hat{n}_{1} & \hat{r}_{1} \hat{n}_{2} & \hat{r}_{1} \hat{n}_{3} \\
\hat{r}_{2} \hat{n}_{1} & \hat{r}_{2} \hat{n}_{2} & \hat{r}_{2} \hat{n}_{3} \\
\hat{r}_{3} \hat{n}_{1} & \hat{r}_{3} \hat{n}_{2} & \hat{r}_{3} \hat{n}_{3}
\end{array}\right]
\end{aligned}
$$

and the stiffness tensor $\mathbf{R}$ in equation (48) with component form

$$
\begin{aligned}
{\left[R_{i j}\right]=} & \frac{2 \mu}{r}(\hat{\mathbf{n}} \cdot \hat{\mathbf{r}})\left[\begin{array}{lll}
1 & 0 & 0 \\
0 & 1 & 0 \\
0 & 0 & 1
\end{array}\right]-\frac{\lambda+2 \mu}{r}\left[\begin{array}{lll}
\hat{n}_{1} \hat{r}_{1} & \hat{n}_{1} \hat{r}_{2} & \hat{n}_{1} \hat{r}_{3} \\
\hat{n}_{2} \hat{r}_{1} & \hat{n}_{2} \hat{r}_{2} & \hat{n}_{2} \hat{r}_{3} \\
\hat{n}_{3} \hat{r}_{1} & \hat{n}_{3} \hat{r}_{2} & \hat{n}_{3} \hat{r}_{3}
\end{array}\right] \\
& +\frac{2 \mu}{r}\left[\begin{array}{lll}
\hat{r}_{1} \hat{n}_{1} & \hat{r}_{1} \hat{n}_{2} & \hat{r}_{1} \hat{n}_{3} \\
\hat{r}_{2} \hat{n}_{1} & \hat{r}_{2} \hat{n}_{2} & \hat{r}_{2} \hat{n}_{3} \\
\hat{r}_{3} \hat{n}_{1} & \hat{r}_{3} \hat{n}_{2} & \hat{r}_{3} \hat{n}_{3}
\end{array}\right] .
\end{aligned}
$$

